\title{
The use of oximetry and a questionnaire in primary care enables exclusion of a subsequent obstructive sleep apnea diagnosis
}

\author{
Timon M. Fabius ${ }^{1,2,3}$ (D) Jeffrey R. Benistant ${ }^{1} \cdot$ Rick G. Pleijhuis $^{4} \cdot$ Job van der Palen ${ }^{3,5} \cdot$ Michiel M. M. Eijsvogel $^{1,2}$
}

Received: 13 November 2018 / Revised: 13 March 2019 / Accepted: 21 March 2019 / Published online: 6 April 2019

(C) The Author(s) 2019

\begin{abstract}
Purpose The study aims to prospectively validate the prognostic value of oximetry alone or combined in a two-step strategy with a questionnaire for the exclusion of obstructive sleep apnea (OSA) in primary care.

Methods A total of 140 subjects with suspected OSA were included from 54 participating primary care practices. All subjects completed the Philips questionnaire and underwent one night of oximetry prior to referral to a sleep center. The prognostic value of two strategies was evaluated against the diagnosis of the sleep center as the gold standard: (1) assume OSA and subsequently refer to a sleep center if the oxygen desaturation index (ODI) is $\geq 5$ and (2) assume OSA and refer to a sleep center if the Philips questionnaire score is $\geq 55 \%$ (regardless of the ODI) or if the Philips questionnaire score is $<55 \%$ and the ODI is $\geq 5$.

Results OSA was diagnosed in the sleep centers in $100(71 \%)$ of the included subjects. Using ODI $\geq 5$ alone resulted in a sensitivity of $99.0 \%$, a specificity of $50.0 \%$, a negative predictive value of $95.2 \%$, and a positive predictive value $83.2 \%$. Using the two-step strategy, oximetry would be performed on $39 \%$ of the subjects. This strategy resulted in a sensitivity of $100 \%$, a specificity of $35.0 \%$, a negative predictive value of $100 \%$, and a positive predictive value of $79.4 \%$.

Conclusions In a Dutch primary care population with a clinical suspicion of OSA and low frequency of cardiovascular comorbidities, the use of oximetry alone or combined in a two-step strategy with a questionnaire enables exclusion of a sleep center diagnosis of OSA.
\end{abstract}

Keywords Screening · Primary care · Obstructive sleep apnea · Oximetry · Questionnaire

Timon M. Fabius and Jeffrey R. Benistant contributed equally to this work.

Electronic supplementary material The online version of this article (https://doi.org/10.1007/s11325-019-01834-2) contains supplementary material, which is available to authorized users.

Timon M. Fabius

T.Fabius@mst.nl

Jeffrey R. Benistant

J.Benistant@mst.nl

Rick G. Pleijhuis

r.g.pleijhuis@gmail.com

Job van der Palen

J.vanderPalen@mst.nl

Michiel M. M. Eijsvogel

M.Eijsvogel@mst.nl
1 Department of Pulmonology, Medisch Spectrum Twente, Onderzoeksbureau Longgeneeskunde, P.O. Box 50000, 7500KA Enschede, The Netherlands

2 Sleep Center, Medisch Spectrum Twente, Enschede, The Netherlands

3 Department of Research Methodology, Measurement and Data Analysis, Faculty of Behavioural, Management and Social Sciences, University of Twente, Enschede, The Netherlands

4 Department of Internal Medicine, Medisch Spectrum Twente, Enschede, The Netherlands

5 Medical School Twente, Medisch Spectrum Twente, Enschede, The Netherlands 


\begin{tabular}{|c|c|}
\hline \multicolumn{2}{|c|}{ Abbreviations } \\
\hline OSA & Obstructive sleep apnea \\
\hline ODI & Oxygen desaturation index \\
\hline AHI & Apnea-hypopnea index \\
\hline AUC & $\begin{array}{l}\text { Area under the receiver operating } \\
\text { characteristics curve }\end{array}$ \\
\hline $95 \% \mathrm{CI}$ & $95 \%$ confidence interval \\
\hline SD & Standard deviation \\
\hline IQR & Interquartile range \\
\hline
\end{tabular}

\section{Introduction}

Obstructive sleep apnea (OSA) is a common sleep disorder that causes patients to stop breathing during sleep. Over the past decades, the hazardous effects of OSA on personal health [1] and society $[2,3]$ have become more and more clear. Still, it is estimated that more than half of all patients suffering from OSA are undiagnosed and therefore untreated [4].

Diagnosing OSA and setting up an appropriate treatment requires specialized care that is generally only available in sleep clinics. In the Netherlands, the number of referrals to sleep clinics for OSA approached 100.000 in 2017 and has increased rapidly over the past few years [5]. Of all patients referred by their general practitioner, up to one third (30\%) eventually does not have OSA upon final poly(somno)graphy [5]. This may be explained by the heterogeneous and often non-specific symptoms of OSA, making it difficult to distinguish OSA from other diagnoses.

Simply increasing the number of referrals to sleep clinics would result in more treated OSA patients, but also in an unacceptable rise in costs due to an associated increase in expensive poly(somno)graphies performed in patients without OSA. In addition, the capacity of sleep clinics is limited, resulting in (extended) waiting lists when the number of referred patients grows. To maximize the number of treated OSA patients without increasing costs or waiting lists, we propose to focus on optimization of the selection process regarding which patients should (not) be referred.

In an attempt to triage referral based on the pre-test probability of OSA, several screening strategies have been developed. Examples include questionnaires (e.g., STOP-BANG $[6,7]$, Berlin [8], Epworth Sleepiness Scale [9]) or a twostep screening strategy (e.g., the Philips questionnaire combined with nasal flow recording [10]). However, although some are suitable for screening in low-prevalence populations, none of these have shown acceptable sensitivity to safely rule out OSA when used in a high-prevalence referral population. In 2016, Kunisaki et al. reported on a prospective observational study in which overnight oximetry was applied to detect OSA in 234 veterans referred for sleep testing [11]. Based on a positive predictive value of 92 to $100 \%$, the authors concluded that overnight oximetry could significantly reduce the number of patients requiring referral for polysomnography.

In this study, we hypothesized that overnight oximetry alone, or combined with a previously published questionnaire in a two-step strategy, could be used to safely rule out OSA in patients visiting their general practitioner with potentially OSA-related complaints, thereby reducing the number of patients requiring referral for sleep testing. We prospectively validated this hypothesis in a high-prevalence population of patients with suspected OSA in the general practice setting.

\section{Methods}

\section{Participants}

This was a prospective observational validation study in 54 general practitioner practices located in the catchment area of the sleep centers of two large teaching hospitals. All adult $(\geq$ 18 years) subjects referred to one of the sleep centers by a participating general practitioner due to suspected OSA were eligible for inclusion. Patients were approached and included by their general practitioner. Subjects who were unable to undergo oximetry or had missing data (i.e., missing questionnaire, oximetry, or sleep center diagnosis) were excluded from the analysis.

All participating subjects provided written informed consent. The study protocol was approved by the Medical Ethical Committee Twente (Enschede, the Netherlands) and registered at the Netherlands Trial Register (www.trialregister.nl, ID: NTR5786).

\section{Measurements}

Included subjects completed an online version of the Philips questionnaire [10] and underwent overnight oximetry using the Nonin WristOx ${ }_{2}{ }^{\mathrm{TM}}$ model 3150 wrist-worn pulse oximeter (Nonin Medical, Inc., Plymouth, MN, USA). The questionnaires were completed without the presence or further explanation of a health care professional. Of the patient characteristics body weight and height were patient reported. Neck circumference was measured at the general practitioner's practice. For the oximetry, subjects were instructed to start the measurement when they went to bed. The oxygen desaturation index (ODI; number of saturation drops $(\geq 3 \%)$ divided by recording time) was automatically obtained from the oximetry data using a custom-build script in Matlab (The Mathworks, Inc., Natick, MA, USA).

All measurements were performed before OSA was diagnosed or excluded in the sleep center. The diagnosis or exclusion of OSA in the sleep center was based on regular care (i.e., symptoms, medical history, physical examination, and poly(somno)graphy). Similar to those of the American 
Academy of Sleep Medicine, the Dutch guideline for diagnosis and treatment of OSA in adults recommends a diagnosis of OSA if poly(somno)graphy results in an apnea-hypopnea in$\operatorname{dex}(\mathrm{AHI}) \geq 15$ or an AHI between 5 and 15 combined with specific symptoms or comorbidities [12, 13]. The poly(somno)graphy data were analyzed according to the American Academy of Sleep Medicine guidelines [14]. Desaturation were defined as a $\geq 3 \%$ decrease from preevent baseline. In polygraphy, hypopneas had to be associated with a desaturation. In polysomnography, hypopneas had to be associated with either a desaturation or an arousal. In case of OSA associated symptoms but a negative polygraphy (i.e., $\mathrm{AHI}<5$ ), a polysomnography was performed (as per AASM and Dutch guidelines). The health care professionals of the sleep centers were unaware of the results of the questionnaire and overnight oximetry.

\section{Statistical analysis}

The primary aim of this study was to validate a predefined strategy to rule out OSA using oximetry alone or combined with the Philips questionnaire in a two-step strategy. For oximetry, an ODI $<5$ has high resemblance with an $\mathrm{AHI}<5$ when measured simultaneously [15]. Therefore, an ODI $<5$ was chosen as cutoff for the oximetry. The Philips questionnaire was originally developed in a population of healthy blueand white-collar workers and results in a score ranging from 0 to $100 \%$. A score below $35 \%$ indicated a low risk on OSA, a score between 35 and $55 \%$ an intermediate risk, and a score of $55 \%$ and above a high risk [10]. The development and validation (along with a full text English version of the questionnaire) are published elsewhere [10]. In the current study, the questionnaire was combined in a two-step strategy by ruling out OSA in those patients with an ODI $<5$ and a score on the Philips questionnaire below $55 \%$ (i.e., those with an ODI $\geq 5$ or a high risk on OSA according to the Philips questionnaire should be referred to a sleep center).

The results of the oximetry alone and the two-step strategy were compared with the diagnosis from the sleep centers using cross-tabulation. Subsequently, sensitivity, specificity, negative predictive value, positive predictive value, and area under the receiver operating characteristics curve (AUC) were calculated. Data were analyzed using SPSS, version 24 (SPSS Inc., Chicago, IL, USA).

A secondary exploratory analysis was performed to identify optimal cutoffs for the questionnaire and oximetry for the exclusion of OSA.

\section{Sample size}

Given the burden of untreated OSA, a high sensitivity is desirable. For this study, a sensitivity of $97 \%$ with a precision of $10 \%$ and a lower boundary of the $95 \%$ confidence interval
(95\% CI) of $90 \%$ was deemed acceptable. To achieve this, 68 subjects with subsequent sleep center diagnosis of OSA should be included. The prevalence of OSA in patients referred to the participating sleep centers is approximately $70 \%$. It was expected that the general practitioners might be keener on referring patients with suspected OSA due to study participation, resulting in a lower prevalence of OSA in those referred. The prevalence in the study population was therefore estimated at 50\%, resulting in 136 subjects needed with complete data. With an estimated dropout of $10 \%$, it was expected that a total of 150 subjects needed to be included.

\section{Results}

Of 164 included subjects, 140 had complete data (see flowchart; Fig. 1). Of these, 119 (85\%) had an ODI $\geq 5$ and 85 $(61 \%)$ a Philips questionnaire score $\geq 55 \%$. OSA was diagnosed in the sleep centers in 100 subjects (71\%). Characteristics of the analyzed subjects are provided in Table 1.

Using an ODI $<5$ alone ruled out OSA in 21 (15\%) subjects of which one did have OSA diagnosed in the sleep center. Cross-tabulation of the use of ODI alone against the sleep center diagnosis is provided in Table 2 . This strategy resulted in a sensitivity of $99.0 \%$ (95\% CI $94.5-100.0 \%)$, a specificity of $50.0 \%$ (95\% CI 33.8-66.2\%), a negative predictive value of 95.2\% (95\% CI 76.2-99.9\%), a positive predictive value of $83.2 \%$ (95\% CI 75.2-89.4\%), a positive likelihood ratio of 1.98 (95\% CI 1.45-2.70), a negative likelihood ratio of 0.02 (95\% CI 0.00-0.14), and a corresponding AUC of 0.75 (95\% CI 0.64-0.85). The subject who was diagnosed with OSA in the sleep center while the screening oximetry resulted in an ODI $<5$ had a score of $98 \%$ on the Philips questionnaire, an ODI of $3 / \mathrm{h}$, and a respiratory event index in the sleep center of $6.4 / \mathrm{h}$ as measured by polygraphy.

The two-step strategy to refer to the sleep center if the Phillips questionnaire is $\geq 55 \%$ or the ODI is $\geq 5$ (i.e., OSA is only excluded if the Philips questionnaire is $<55 \%$ and ODI <5) ruled out OSA in $14(10 \%)$ subjects of which none had OSA diagnosed in the sleep centers. Crosstabulation of the two-step strategy against the sleep center diagnosis is provided in Table 3 . This strategy resulted in a sensitivity of $100 \%$ (95\% CI 96.3-100.0), a specificity of $35.0 \%$ (95\% CI 20.6-51.7\%), a negative predictive value of $100 \%$ (95\% CI $76.8-100.0 \%$ ), a positive predictive value of $79.4 \%$ (95\% CI 71.2-86.1\%), a positive likelihood ratio of 1.54 (95\% CI 1.23-1.93), a negative likelihood ratio of 0.00 (95\% CI not applicable), and a corresponding AUC of 0.68 (95\% CI 0.57-0.79).

The explorative analysis showed that an optimal combination of sensitivity and specificity could be achieved by assuming OSA (and subsequently refer to the sleep center) if one of 
Fig. 1 Flowchart of the included subjects

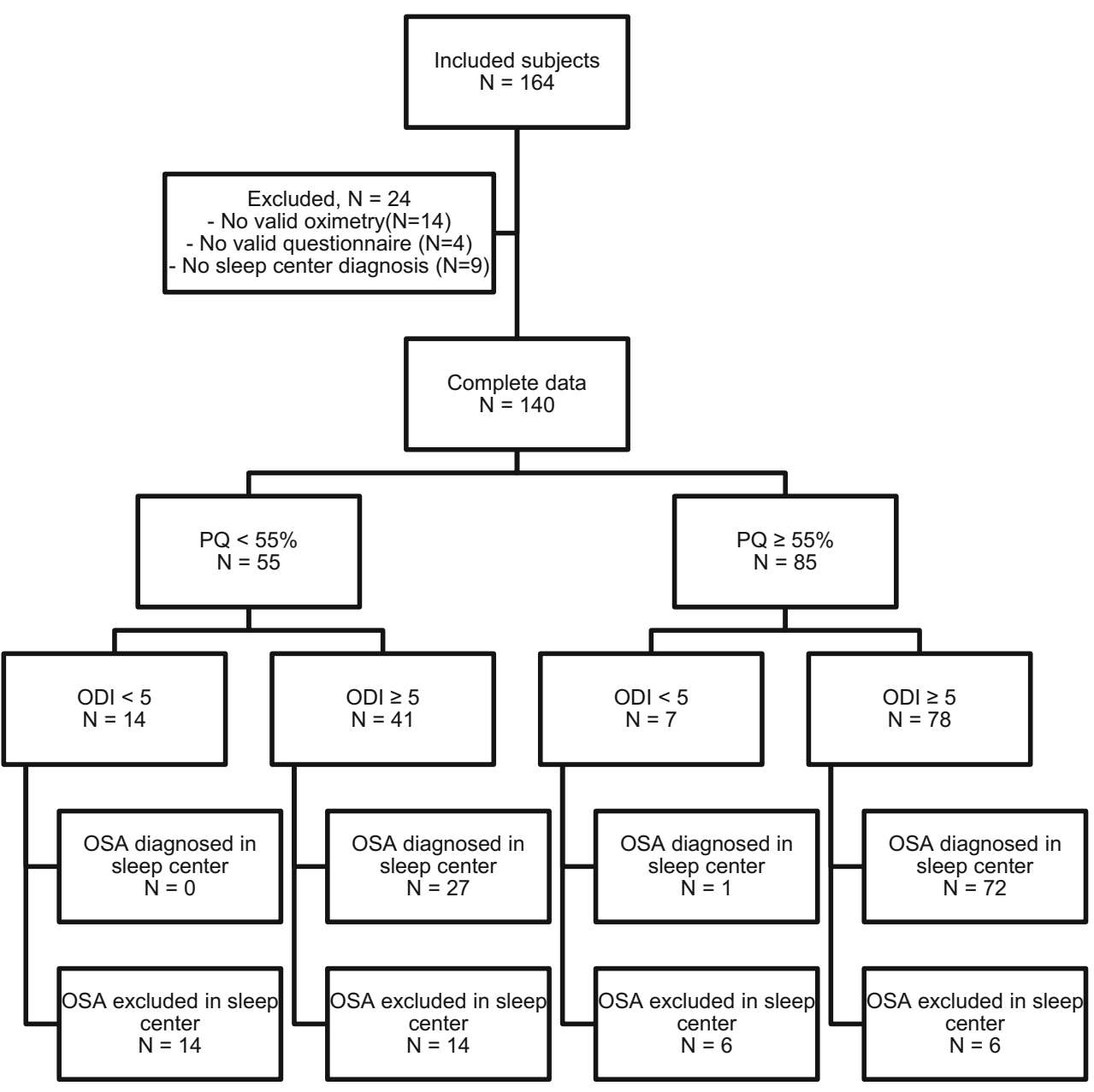

the following three conditions applied: (1) the Philips questionnaire was $\geq 92 \%$, or (2) the ODI rounded to the nearest integer was $\geq 10$, or (3) the rounded ODI was between 5 and 10 and the Philips questionnaire was $\geq 46.5 \%$. Crosstabulation of these optimized cutoffs versus the final diagnosis of the sleep centers is provided in Table 4. These cutoffs would result in a sensitivity of $99.0 \%$ (95\% CI 94.6$100.0 \%$ ), a specificity of $65.0 \%$ (95\% CI $48.3-79.4 \%$ ), a negative predictive value of $96.3 \%$ (95\% CI $81.0-99.9 \%$ ), a positive predictive value of $87.6 \%$ (95\% CI $80.1-93.1 \%$ ), a positive likelihood ratio of 2.83 (95\% CI 1.85-4.32), a negative likelihood ratio of 0.02 (95\% CI $0.00-0.11)$, and a corresponding AUC of 0.82 (95\% CI 0.73-0.91).

\section{Discussion}

The primary aim of this study was to validate the use of oximetry alone or combined with a questionnaire to exclude OSA in primary care. The results show that both pre-defined strategies have a high sensitivity (slightly higher for the twostep strategy) and moderate specificity (slightly higher for oximetry alone). The strength of oximetry alone is the higher specificity, which would significantly decrease the number of subjects referred for further workup. However, this strategy seems to erroneously exclude OSA in approximately $1 \%$ of the subjects with subsequently diagnosed OSA when referred to a sleep center, whereas no OSA case was missed using the two-step strategy. Furthermore, if the two-step strategy is applied, oximetry would only be needed in $39 \%$ of the patients. On the other hand, the two-step strategy excludes OSA in fewer subjects (10 versus 15\%). Moreover, the respiratory event index resulting from the sleep center analysis in the one false-negative subject in the oximetry-only strategy was only slightly higher than the cutoff of five events per hour. This may have been caused by the known night-to-night variation in OSA severity [16]. Both strategies seem to have their strengths and the exact costs will strongly depend on the local costs of an oximetry reading and the local health care system. An extensive cost-effectiveness analysis elaborating on the abovementioned screening strategies was recently published elsewhere [17].

The exploratory analysis resulted in cutoffs that can substantially increase the specificity of the two-step strategy with only a limited decrease in sensitivity. This would add substantially to the clinical applicability of the strategy. However, it 
Table 1 Characteristics of the included subjects

$\begin{array}{lll}\text { Total }(N=140) & \begin{array}{l}\text { OSA diagnosed in sleep } \\ \text { center }(N=100)\end{array} & \begin{array}{l}\text { OSA excluded in sleep } \\ \text { center }(N=40)\end{array}\end{array} \quad P$ value

\begin{tabular}{|c|c|c|c|c|}
\hline \multicolumn{5}{|l|}{ General characteristics } \\
\hline Age, years (SD) & $49.3(13.7)$ & $52.4(12.6)$ & $41.4(13.1)$ & $<0.001$ \\
\hline Males, $N(\%)$ & $101(72.1)$ & $78(78.0)$ & $23(57.5)$ & 0.02 \\
\hline Weight, kg (SD) & $96.3(18.0)$ & $99.4(18.2)$ & $88.6(15.0)$ & 0.001 \\
\hline Body mass index, $\mathrm{kg} / \mathrm{m}^{2}$ (IQR) & $29.4(25.7-33.3)$ & $30.7(26.4-34.4)$ & $26.7(24.7-29.4)$ & $<0.001$ \\
\hline Heart failure, $N(\%)$ & $1(0.7)$ & $1(1.0)$ & $0(0.0)$ & 1.00 \\
\hline Cardiac ischemia, $N(\%)$ & $7(5.0)$ & $6(6.0)$ & $1(2.5)$ & 0.67 \\
\hline Atrial fibrillation, $N(\%)$ & $9(6.4)$ & $9(9.0)$ & $0(0.0)$ & 0.06 \\
\hline Hypertension, $N(\%)$ & $35(25.0)$ & $33(33.0)$ & $2(5.0)$ & 0.001 \\
\hline Hypercholesterolemia, $N(\%)$ & $12(8.6)$ & $10(10.0)$ & $2(5.0)$ & 0.509 \\
\hline Diabetes mellitus, $N(\%)$ & $12(8.6)$ & $11(11.0)$ & $1(2.5)$ & 0.18 \\
\hline Malignancy, $N(\%)$ & $6(4.3)$ & $6(6.0)$ & $0(0.0)$ & 0.18 \\
\hline Hypothyroidism, $N(\%)$ & $9(6.4)$ & $4(4.0)$ & $5(12.5)$ & 0.12 \\
\hline Stroke, $N(\%)$ & $5(3.6)$ & $4(4.0)$ & $1(2.5)$ & 1.00 \\
\hline COPD, $N(\%)$ & $3(2.1)$ & $2(2.0)$ & $1(2.5)$ & 1.00 \\
\hline \multicolumn{5}{|l|}{ Measured by the general practitioner } \\
\hline Neck circumference, $\mathrm{cm}(\mathrm{SD})$ & $40.6(3.9)$ & $41.5(3.6)$ & $38.4(3.6)$ & $<0.001$ \\
\hline Oxygen desaturation index (IQR) & $11.9(6.1-22.1)$ & $16.2(10.6-25.4)$ & $5.1(3.1-7.0)$ & $<0.001$ \\
\hline Oxygen desaturation index $\geq 5, N(\%)$ & $119(85.0)$ & $99(99.0)$ & $20(50.0)$ & $<0.001$ \\
\hline Philips questionnaire, \% (IQR) & $70.1(46.6-93.1)$ & $89.2(51.8-94.8)$ & $46.6(28.0-65.8)$ & $<0.001$ \\
\hline Philips questionnaire $\geq 55 \%, N(\%)$ & $85(60.7)$ & $73(73.0)$ & $12(30.0)$ & $<0.001$ \\
\hline \multicolumn{5}{|l|}{ Measured at the sleep center } \\
\hline Epworth Sleepiness Scale (SD, $N=125)$ & $7.3(4.5)$ & $7.6(4.7)$ & $6.6(4.1)$ & 0.30 \\
\hline Apnea-hypopnea index (IQR) & $13.6(6.3-23.8)$ & $18.2(12.9-31.8)$ & $3.3(2.5-4.8)$ & $<0.001$ \\
\hline Apnea index (IQR) & $1.0(0.4-5.5)$ & $2.8(0.5-9.2)$ & $0.3(0.0-0.7)$ & $<0.001$ \\
\hline Hypopnea index (IQR) & $10.1(4.7-16.6)$ & $13.7(8.6-18.7)$ & $2.8(1.5-4.3)$ & $<0.001$ \\
\hline Apnea-hypopnea index $5-15, N(\%)$ & $47(33.6)$ & $38(38.0)$ & $9(22.5)$ & 0.079 \\
\hline Apnea-hypopnea index 15-30, N(\%) & $35(25.0)$ & $35(35.0)$ & $0(0.0)$ & $<0.001$ \\
\hline Apnea-hypopnea index $\geq 30, N(\%)$ & $27(19.3)$ & $27(27.0)$ & $0(0.0)$ & $<0.001$ \\
\hline
\end{tabular}

Data are presented as mean with standard deviation (SD), median with interquartile range (IQR), or number with corresponding percentage

would require oximetry recordings in all subjects, whereas the predefined cutoffs would only require oximetry in those with a low to moderate score on the Philips questionnaire. Again, whether the added costs of the oximetry reading will outweigh the saved costs of the prevented sleep center referrals strongly depends on the local health care system. More importantly, the optimized cutoffs should be validated prospectively before they can be used in clinical practice.

The findings of this study confirm that a referral to a sleep center for the diagnosis of OSA can be omitted in primary care using oximetry alone or a two-step strategy combining oximetry and a questionnaire. A recent study in Spain showed that the workup and management of OSA in primary care was non-inferior to the workup and management of OSA in a specialized sleep center [18]. Similar results were reported earlier from a study in Australia [19]. We aimed to validate the exclusion of OSA in primary care rather than its confirmation and management. Although the mentioned trials suggest that management is also feasible, it is important to note that both studies only included uncomplicated patients and management was performed by trained nurses. In our study, only subjects who were unable to undergo oximetry or refused informed consent were excluded. Furthermore, no strict inclusion criteria were applied. The findings of our study seem therefore applicable to a broader population. It should be noted, however, that our study was not powered to validate the proposed strategies in subpopulations (e.g., subjects with several significant comorbidities).

With the right inclusion and exclusion criteria one might argue that an aberrant oximetry recording alone might be sufficient to start a CPAP trial. However, the use of oximetry alone (rather than a poly(somno)graphy recording) has inherent limitations. Foremost, an oximetry reading provides much less information than a poly(somno)graphy recording. For 
Table 2 Cross-tabulation of the use of oximetry alone versus the diagnosis of the sleep centers

\begin{tabular}{lclr}
\hline & OSA diagnosed & OSA excluded & Total \\
\hline ODI $\geq 5$ & 99 & 20 & 119 \\
ODI $<5$ & 1 & 20 & 21 \\
Total & 100 & 40 & 140 \\
\hline
\end{tabular}

ODI oxygen desaturation index

instance, oximetry will not allow to differentiate between central and obstructive events or if there is a significant positional dependence. Both might enable (or even require) other treatment strategies than plain CPAP. Nevertheless, oximetry alone may be sufficient for a selected (sub) group of patients. The key will lie in the way this group of patients is selected. A large Australian study already investigated the use of full polysomnography versus polygraphy versus oximetry as the basis for the management of OSA [20]. The results showed that oximetry alone (compared to polysomnography) resulted in shorter CPAP usage and less improvement in reported sleep apnea symptoms. Though subjects with significant comorbidities were excluded, all ranges of the ODI were used for management decisions. One might argue that the combination of a high ODI (e.g., $>30$ or $40 / \mathrm{h}$ ), the absence of significant comorbidities (such as heart failure and neuromuscular conditions), and a high clinical probability of OSA (according to a sleep expert) might enable selection of those patients in whom CPAP can be started without additional testing. If (as was done in our study) the ODI is calculated automatically, we recommend manual affirmation of the automated analysis. In addition, future prospective studies are needed to investigate this interesting hypothesis. Our study has some limitations. First, the clinical diagnosis rather than a test result was used as reference standard. This choice was based on the recent reports indicating a very high prevalence of an increased $\mathrm{AHI}$ in the general population of which many do not have any symptoms [21]. Although the debate on whether all asymptomatic individuals with an increased AHI can be left untreated is still ongoing, we feel that the diagnosis of OSA should be based on the combination of AHI with symptoms. This is partly reflected in our results, as OSA was not diagnosed in 10 subjects with an elevated AHI. A second point worthy of note is the number of included subjects. Based on

Table 3 Cross-tabulation of the two-step strategy alone versus the diagnosis of the sleep centers

\begin{tabular}{lclc}
\hline & OSA diagnosed & OSA excluded & Total \\
\hline $\mathrm{PQ} \geq 55 \%$ or $\mathrm{ODI} \geq 5$ & 100 & 26 & 126 \\
$\mathrm{PQ}<55 \%$ and $\mathrm{ODI}<5$ & 0 & 14 & 14 \\
Total & 100 & 40 & 140 \\
\hline
\end{tabular}

PQ Philips questionnaire, ODI oxygen desaturation index
Table 4 Cross-tabulation of the optimal (according to the post hoc analysis) two-step strategy versus the diagnosis of the sleep centers

\begin{tabular}{lrlr}
\hline & OSA diagnosed & OSA excluded & Total \\
\hline Optimal strategy positive & 99 & 14 & 113 \\
Optimal strategy negative & 1 & 26 & 27 \\
Total & 100 & 40 & 140 \\
\hline
\end{tabular}

This strategy was deemed positive (i.e., OSA is assumed likely and a subject should be referred to a sleep center) if (1) the Philips questionnaire was $\geq 92 \%$, or (2) the ODI rounded to the nearest integer was $\geq 10$, or (3) the rounded ODI was between 5 and 10 and the Philips questionnaire was $\geq 46.5 \%$

OSA obstructive sleep apnea, ODI oxygen desaturation index

the sample size analysis, 68 subjects in whom OSA was subsequently diagnosed should have been included. However, 100 were included. This was mainly caused by a delay between inclusion (and study measurements) and the time of diagnosis in the sleep centers. Another noteworthy element is that a negative screening result does not exclude the presence of other sleep pathology. If implemented in clinical practice, it should be emphasized that the screening strategies are only capable of excluding OSA and subsequent referral to a sleep center for some other sleep pathology might still be useful. The addition of other (non-OSA) sleep-related questions might help the general practitioner to recognize such cases. As an example, the Athens Insomnia Scale [22] is already incorporated in the Philips questionnaire [10].

Finally, although it was not an exclusion criterion, the prevalence of comorbidities that might significantly influence the results of an oximetry reading such as COPD, heart failure, or neuromuscular disorders was low in the study population. Furthermore, the study population was predominantly male. This may reflect clinical practice but there are significant differences in OSA pathophysiology and presentation between males and females [23]. This may influence the accuracy of diagnostic strategies. We did not observe large differences in accuracy of the three presented strategies between males and females (see supplementary data). However, our study was not powered to prove differences in diagnostic accuracy between males and females. This precludes a firm conclusion on the use of oximetry for OSA screening in females and subjects with specific comorbidities. Future studies should address the accuracy of oximetry (combined with a questionnaire) in specific comorbidities and females.

Summarizing, in a Dutch primary care population with a clinical suspicion of OSA and low frequency of cardiovascular comorbidities, the use of oximetry alone or combined in a two-step strategy with a questionnaire enables exclusion of a sleep center diagnosis of OSA. The two-step strategy with oximetry only in those with a low probability for OSA (based on the Philips questionnaire) results in a higher sensitivity but 
lower specificity when compared with oximetry alone. The two-step strategy may be more cost-effective as fewer oximetry recordings would be needed.

Acknowledgements The authors would like to gratefully acknowledge Annemarijn Quittner, Karst Boersma, Marijn Kroese, Charlotte Hoogenkamp, Wieke Wolthuis, Susan Mensink, Joanne Berger, Romy Schröer, and the staff of all participating general practitioner practices for their assistance in patient inclusion and study measurements.

Funding This study was financially supported by an unrestricted research grant from the Pioneers in Health Care Innovation Fund, established by the University of Twente, Medisch Spectrum Twente, ZiekenhuisGroep Twente, and Menzis Health Insurance cooperation.

\section{Compliance with ethical standards}

Institution This study was performed in 54 general practitioner practices located in the catchment area of the sleep centers of two teaching hospitals (ZiekenhuisGroep Twente, Almelo, the Netherlands and Medisch Spectrum Twente, Enschede, the Netherlands). Study conception, design, supervision, and data analysis were performed at the Department of Pulmonology, Medisch Spectrum Twente, Enschede, the Netherlands.

Author statement All authors have read and approved the current manuscript.

Conflict of interests JB, RP, and ME report shares in DiagnOSAS B.V., a company aiming to facilitate screening on OSA in primary care. TF and JvdP declare they have no conflict of interests.

Study registration The study was registered at the Netherlands Trial Register (DiagnOSAS as a screening tool for obstructive sleep apnea syndrome in the primary care setting, http://www.trialregister.nl/trialreg/ admin/rctview.asp?TC=5786, ID: NTR5786).

Ethical approval All participating subjects provided written informed consent. The study protocol was approved by the Medical Ethical Committee Twente (Enschede, the Netherlands).

Open Access This article is distributed under the terms of the Creative Commons Attribution 4.0 International License (http:// creativecommons.org/licenses/by/4.0/), which permits unrestricted use, distribution, and reproduction in any medium, provided you give appropriate credit to the original author(s) and the source, provide a link to the Creative Commons license, and indicate if changes were made.

\section{References}

1. McNicholas WT, Bonsigore MR, Bonsignore MR, Management Committee of EU COST ACTION B26 (2007) Sleep apnoea as an independent risk factor for cardiovascular disease: current evidence, basic mechanisms and research priorities. Eur. Respir. J. 29: $156-178$

2. Hartenbaum N, Collop N, Rosen IM, Phillips B, George CFP, Rowley JA, Freedman N, Weaver TE, Gurubhagavatula I, Strohl K, Leaman HM, Moffitt GL, Rosekind MR (2006) Sleep apnea and commercial motor vehicle operators: statement from the joint task force of the American College of Chest Physicians, American College of Occupational and Environmental Medicine, and the National Sleep Foundation. J. Occup. Environ. Med. 48:S4-S37

3. Jennum P, Kjellberg J (2011) Health, social and economical consequences of sleep-disordered breathing: a controlled national study. Thorax 66:560-566

4. Young T, Palta M, Dempsey J, Peppard PE, Nieto FJ, Hla KM (2009) Burden of sleep apnea: rationale, design, and major findings of the Wisconsin sleep cohort study. WMJ 108:246-249

5. Kantar Public, Dutch Apnea Society (2018) Diagnosis and treatment of sleep apnea in sleep clinics 2018 (In Dutch, original title: "Diagnose en behandeling van apneu in slaapklinieken 2018")

6. Chung F, Yegneswaran B, Liao P, Chung SA, Vairavanathan S, Islam S, Khajehdehi A, Shapiro CM (2008) STOP questionnaire: a tool to screen patients for obstructive sleep apnea. Anesthesiology 108:812-821

7. Chung F, Subramanyam R, Liao P, Sasaki E, Shapiro C, Sun Y (2012) High STOP-Bang score indicates a high probability of obstructive sleep apnoea. Br. J. Anaesth. 108:768-775

8. Netzer NC, Stoohs RA, Netzer CM, Clark K, Strohl KP (1999) Using the Berlin questionnaire to identify patients at risk for the sleep apnea syndrome. Ann. Intern. Med. 131:485-491

9. Rosenthal LD, Dolan DC (2008) The Epworth Sleepiness Scale in the identification of obstructive sleep apnea. J. Nerv. Ment. Dis. 196:429-431

10. Eijsvogel MM, Wiegersma S, Randerath W, Verbraecken J, WegterHilbers E, van der Palen J (2016) Obstructive sleep apnea syndrome in company workers: development of a two-step screening strategy with a new questionnaire. J. Clin. Sleep Med. 12:555-564

11. Kunisaki KM, Bohn OA, Wetherbee EE, Rector TS (2016) Highresolution wrist-worn overnight oximetry has high positive predictive value for obstructive sleep apnea in a sleep study referral population. Sleep Breath. 20:583-587

12. Epstein LJ, Kristo D, Strollo PJ Jr, Friedman N, Malhotra A, Patil SP, Ramar K, Rogers R, Schwab RJ, Weaver EM, Weinstein MD, Adult Obstructive Sleep Apnea Task Force of the American Academy of Sleep Medicine (2009) Clinical guideline for the evaluation, management and long-term care of obstructive sleep apnea in adults. J. Clin. Sleep Med. 5:263-276

13. Eijsvogel MM, Schimsheimer RJ, Vos PJE, de Vries N, Raats CJI, Trooster R (2009) CBO Richtlijn diagnostiek en behandeling van het obstructief slaap apneu syndroom bij volwassenen (Dutch guideline for diagnosis and treatment of OSA in adults). Van Zuiden Communication, Alphen aan den Rijn

14. Berry RB, Budhiraja R, Gottlieb DJ, Gozal D, Iber C, Kapur VK, Marcus CL, Mehra R, Parthasarathy S, Quan SF, Redline S, Strohl KP, Davidson Ward SL, Tangredi MM, American Academy of Sleep Medicine (2012) Rules for scoring respiratory events in sleep: update of the 2007 AASM manual for the scoring of sleep and associated events. Deliberations of the sleep apnea definitions task force of the American Academy of sleep medicine. J. Clin. Sleep Med. 8:597-619

15. Fabius TM, Benistant JR, Bekkedam L, van der Palen J, de Jongh FHC, Eijsvogel MMM (2018) Validation of the oxygen desaturation index in the diagnostic workup of obstructive sleep apnea. Sleep Breath. 23(1):57-63

16. Stöberl AS, Schwarz EI, Haile SR, Turnbull CD, Rossi VA, Stradling JR, Kohler M (2017) Night-to-night variability of obstructive sleep apnea. J. Sleep Res. 26(6):782-788 1-7

17. Geessinck FAJ, Pleijhuis RG, Mentink RJ, van der Palen J, Koffijberg H (2018) Cost-effectiveness analysis of the DiagnOSAS screening tool compared with polysomnography diagnosis in Dutch primary care. J. Clin. Sleep Med. 14:1005-1015

18. Sánchez-Quiroga MÁ, Corral J, Gómez-de-Terreros FJ, CarmonaBernal C, Asensio-Cruz MI, Cabello M, Martínez-Martínez MÁ, Egea CJ, Ordax E, Barbe F, Barca J, Masa JF, Fernández-Zapata G, 
Sánchez-Armengol Á, Caballero-Eraso C, Cantalejo O, Ocon R, Álvarez-Ruiz de Larrinaga A, Azpiazu M, Alonso-Alvarez ML, Tobajas L, Rubio M, González-Espárrago A, Lebrero-Ferreiro R, Hermosin-Bono T, Juan-Armas J, Crespo-Fidalgo P, de-la-Llama MH, Vega-Dominguez ME, Sánchez-Armengol Á, AlonsoMartínez ML, Álvarez-Ruiz de Larrinaga A, Fernández-Zapata G, Caballero-Eraso C, Cantalejo O, Ocon R, Azpiazu M, Tobajas L, Rubio M, González-Espárrago A, Lebrero-Ferreiro R, HermosinBono T, Juan-Armas J, Crespo-Fidalgo P, de-la-Llama MH, VegaDominguez ME (2018) Primary care physicians can comprehensively manage sleep apnea patients: a non-inferiority randomized controlled trial. Am. J. Respir. Crit. Care Med. 198:648-656

19. Chai-Coetzer CL, Antic NA, Rowland LS, Reed RL, Esterman A, Catcheside PG, Eckermann S, Vowles N, Williams H, Dunn S, McEvoy RD (2013) Primary care vs specialist sleep center management of obstructive sleep apnea and daytime sleepiness and quality of life: a randomized trial. JAMA 309:997-1004

20. Chai-Coetzer CL, Antic NA, Hamilton GS, McArdle N, Wong K, Yee BJ, Yeo A, Ratnavadivel R, Naughton MT, Roebuck T, Woodman R, McEvoy RD (2017) Physician decision making and clinical outcomes with laboratory polysomnography or LimitedChannel sleep studies for obstructive sleep apnea: a randomized trial. Ann. Intern. Med. 166:332-340

21. Heinzer R, Vat S, Marques-Vidal P, Marti-Soler H, Andries D, Tobback N, Mooser V, Preisig M, Malhotra A, Waeber G, Vollenweider P, Tafti M, Haba-Rubio J (2015) Prevalence of sleep-disordered breathing in the general population: the HypnoLaus study. Lancet Respir. Med. 3:310-318

22. Soldatos CR, Dikeos DG, Paparrigopoulos TJ (2000) Athens insomnia scale: validation of an instrument based on ICD-10 criteria. J. Psychosom. Res. 48:555-560

23. Theorell-Haglöw J, Miller CB, Bartlett DJ, Yee BJ, Openshaw HD, Grunstein RR (2018) Gender differences in obstructive sleep apnoea, insomnia and restless legs syndrome in adults - what do we know? A clinical update. Sleep Med. Rev. 38:28-38

Publisher's note Springer Nature remains neutral with regard to jurisdictional claims in published maps and institutional affiliations. 